\title{
Synthesis and spectral properties of tetrapyrazinoporphyrazinato metal complexes derived from a sterically hindered phenol
}

\author{
Fadi Ibrahim and Saad Makhseed* \\ Department of Chemistry, University of Kuwait, P.O.Box 5969, Safat-13060, State of Kuwait \\ E-mail: smakhseed@kuc01.kuniv.edu.kw
}

Dedicated to Prof. Nouria Al-Awadi on the occasion of her $55^{\text {th }}$ anniversary

\begin{abstract}
The synthesis of metal-containing tetrapyrazinoporphyrazines (azaphthalocyanines) bearing eight bulky substituents at the peripheral positions is described. The key precursor is prepared using a nucleophilic aromatic substitution reaction between a sterically hindered phenol and 5,6dichloropyrazine-2,3-dicarbonitrile. Thus, azaphthalocyanine (AzaPc) derived from 2,6isopropylphenol possesses substituents that are forced by steric constraints to adopt a non-planar conformation which drastically enhances the solubility of the macrocycles and inhibits efficient cofacial interaction of the macrocycles even in the solid state as evaluated by UV-Vis and ${ }^{1} \mathrm{H}$ NMR spectroscopic techniques. The intense absorption in the red visible region and photostability of these highly soluble and non-aggregated complexes enhance the dynamic activity and make these complexes potentially suitable for PDT.
\end{abstract}

Keywords: Aazaphthalocyanine; aggregation; spin coated films

\section{Introduction}

Phthalocyanine $(\mathrm{Pc})$ and its derivatives are of interest due to their outstanding electronic and optical properties. In addition to their extensive role as colorants they have found application in nonlinear optics, as liquid crystalline electronic charge carriers, photoconductors, optical data storage, solar energy conversion, photodynamic cancer therapy, catalysis and as the active component of gas sensors. ${ }^{1,2}$ The extended planar, four-fold symmetry and stability also makes the phthalocyanine macrocycle an excellent building block for use in supramolecular ${ }^{3}$ and polymer chemistry. 4

Analogues of phthalocyanine in which nitrogen replaces some of the carbons within the four fused benzo-substituents of the phthalocyanine, possess significantly different physical properties including color, oxidation potential and stability, which may be beneficial for some of 
these applications. ${ }^{5,6}$ Hence, 1,4,8,11,15,18,22,25-octaazaphthalocyanine (AzaPc), often termed tetrapyrazinoporphyrazine in the literature, and its substituted derivatives have been investigated extensively for applications as photodynamic therapeutics, ${ }^{7-9}$ colorants, ${ }^{10}$ catalysts, ${ }^{11}$ liquid crystals, $^{12-14}$ non-linear optical materials ${ }^{15}$ and as a red fluorophore. ${ }^{10,16,17}$ This latter application is of particular interest as the major phthalocyanine fluorescence band falls in the Near-IR region and is largely invisible to the human eye whereas AzaPc has a very strong fluorescence band in the visible spectrum. Due to their extended planar $\pi$-conjugated systems, AzaPcs exhibit a high tendency for aggregation which decreases solubility and hinders purification and characterization. Therefore, such unfavorable properties of these macrocyclic compounds greatly reduces their efficiency in $\mathrm{PDT}^{18}$ and other applications. ${ }^{19,20}$ Controlling the morphology of the azaphthalocyanine-based materials affects their performance in the above mentioned applications. For example, molecular self-association leads to major modification of the physicochemical properties, in particular, the principle Q-band in the visible region of the spectrum (e.g. Q-band; $\lambda_{\max } \sim 630 \mathrm{~nm}$ whereas for Pc $\lambda_{\max } \sim 680 \mathrm{~nm}$ ) of the AzaPc chromophore is broadened and blue-shifted due to the effect of intermolecular exciton interactions. Other consequences of self-association are quenching of fluorescence that reduces the ability of the macrocycle to generate the singlet oxygen which is the active agent in photodynamic cancer therapy. Therefore, it is of interest to induce the macrocyclic units to be isolated from each other within the azaphthalocyanine material and consequently act as a solid solution.

Recently we found that the presence of bulky phenoxy substituents prohibits cofacial selfassociation of the phthalocyanine macrocycle even within solid thin films and can result in the formation of a remarkable nanoporous cubic crystal. ${ }^{21,22}$ Therefore, we applied this successful strategy to prepare novel octaazaphthalocyanine derivatives functionalized with eight peripheral 2,6-diisopropyl-4-nitrophenoxy substituents. ${ }^{1} \mathrm{H}$ NMR and UV/Vis spectroscopic techniques were used to explore the influence of such bulky groups on the structure of the octaazaphthalocyanine-containing bulk material.

\section{Results and Discussion}

\section{Synthesis}

The synthetic route used for obtaining the zinc (AzaPc 2) and cobalt (AzaPc 3) containing complexes is shown in Scheme 1. This route is based on the starting precursors, 5,6-dichloropyrazine-2,3-dicarbonitrile $^{23}$ and 2,6-diisopropyl-4-nitrophenol ${ }^{24}$ which were prepared from commercially available materials following previously described procedures. 


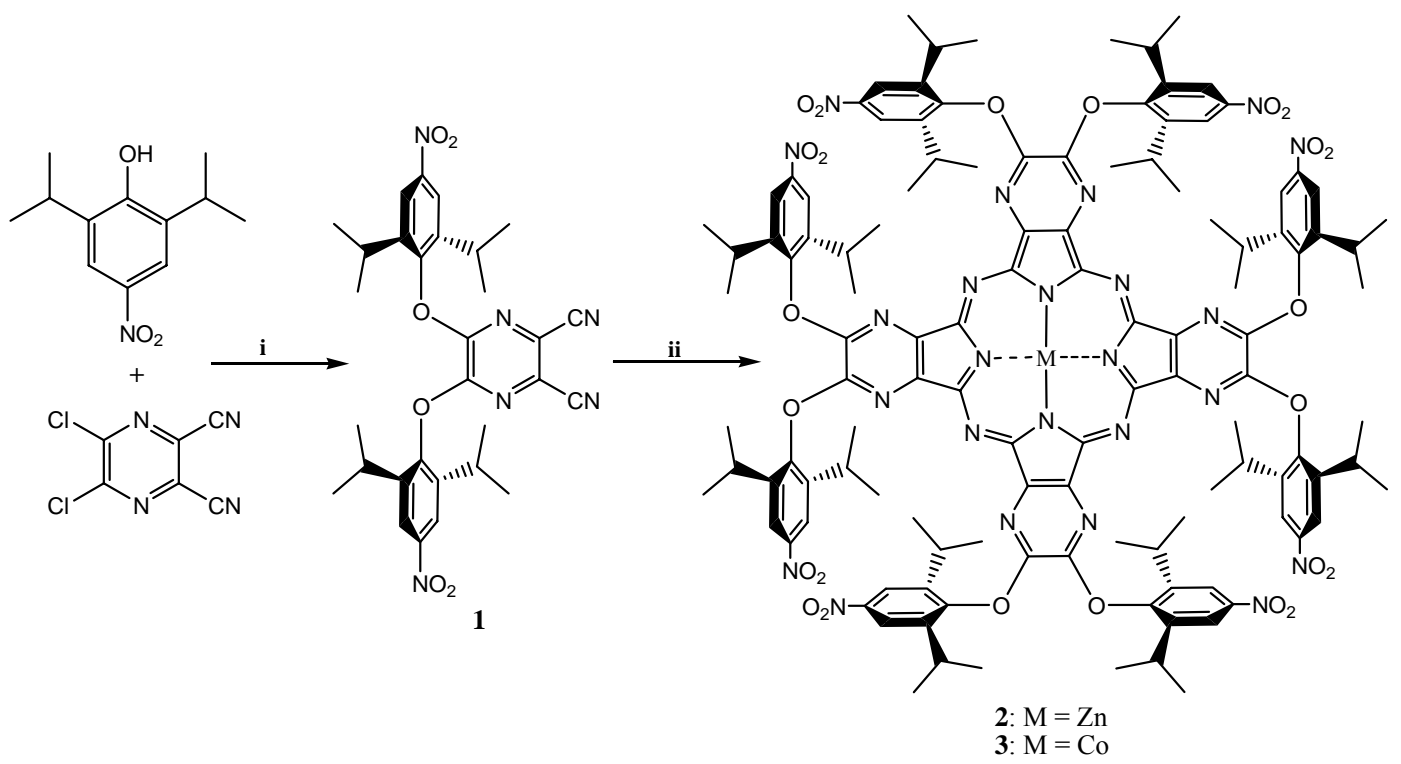

Scheme 1. The synthesis of azaphthalocyanine derivadives. Reagents and conditions: i.dioxane, $\mathrm{NaH}, 100^{\circ} \mathrm{C}$, ii. Quinoline, $160^{\circ} \mathrm{C}$, appropriate anhydrous metal salt.

An aromatic nucleophilic substitution reaction ${ }^{25}$ between 5,6-dichloro-pyrazine-2,3dicarbonitrile and 2,6-diisopropyl-4-nitrophenol in dry dioxane and $\mathrm{NaH}$ at $100{ }^{\circ} \mathrm{C}$ for $24 \mathrm{~h}$ gave the key intermediate 5,6-bis(2,6-diisopropyl-4-nitrophenoxy)pyrazine-2,3-dicarbonitrile $\mathbf{1}$. A milder base catalyzed-reaction $\left(\mathrm{K}_{2} \mathrm{CO}_{3}\right)$ failed to provide the target compound $\mathbf{1}$ in spite of the strong electron-withdrawing ability of the pyrazine-2,3-dicarbonitrile moiety which makes the chlorine atoms labile. Analytical and spectroscopic analysis of $\mathbf{1}$ were consistent with the structure. In the IR spectrum, the absorption at $2220 \mathrm{~cm}^{-1}$ is characteristic of the -CN groups.

The syntheses of AzaPc 2 and AzaPc 3 depended on metal ion template reactions of the dinitrile derivative 1 in quinoline at $160{ }^{\circ} \mathrm{C}$ using anhydrous zinc acetate, or cobalt chloride respectively. ${ }^{26}$ Column chromatography over silica gel was employed using chloroform as eluent to obtain the appropriate metallated AzaPcs $(2,3)$ in good isolated yields $(50-55 \%)$ as compared with other methods. ${ }^{27}$ The high solubility of the intensely green products $(2,3)$ in most organic solvents (e.g. DCM, THF, $\mathrm{CHCl}_{3}$ ) due to the effect of the eight phenoxy groups, probably contributes significantly to the observed good yields. The structure and purity of AzaPc (2, 3) were confirmed by H NMR, UV/Visible, matrix-assisted laser desorption ionization (MALDI) mass spectroscopy, together with elemental analysis. Analysis using MALDI-MS gave, in each case, a cluster of molecular ion peaks consistent with their molecular formulas. The UV/Visble absorption spectra of $(2,3)$ complexes in solution show the characteristic metal ion containing the octaazaphthalocyanine Q-band at $610-630 \mathrm{~nm}$. The percentage weight changes as a function of temperature are shown in Figure 1. From the thermograms it was found that both complexes are highly stable in the temperature range of $300-320{ }^{\circ} \mathrm{C}$ and that above this, extensive decomposition occurs at temperatures between $350-420{ }^{\circ} \mathrm{C}$. Therefore, the stability of 
the prepared Aza-complexes $(2,3)$ is similar to other aza-analogues of phthalocyanines which generally decompose without melting upon heating at high temperature $\left(>300^{\circ} \mathrm{C}\right)$.

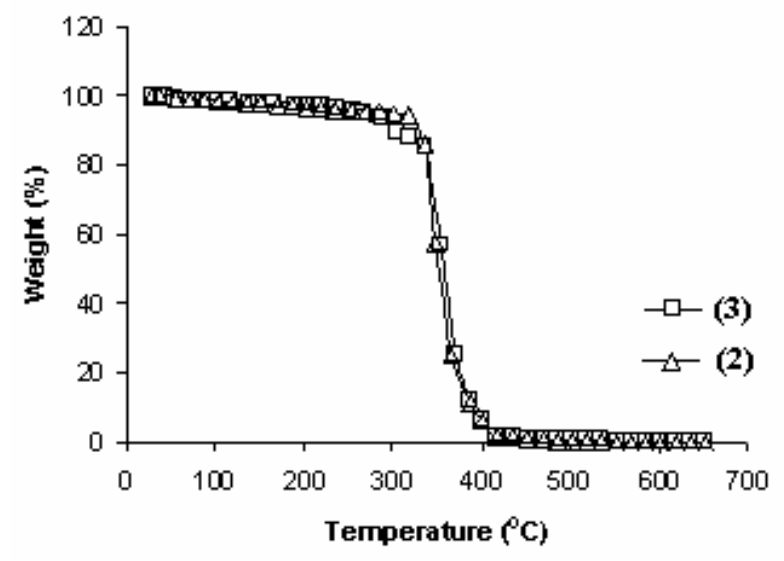

Figure 1. Thermogravimetric curves for AzaPc complexes (2 and 3).

\section{Material properties}

As mentioned in the introduction, the self-association of azaphthalocyanine molecules is apparent even in dilute solution in which the macrocyclic molecules tend to form dimers, trimers and higher oligomers. ${ }^{28}$ This aggregation behavior can considerably affect their properties and limit their uses in many optical and electronic applications. For example, cofacial exciton interactions result in the quenching of the photochemically excited state of AzaPc and interfere with the formation of singlet oxygen. ${ }^{29}$ Thus, our aim was successfully achieved, to avoid the cofacial self-association by introducing eight bulky phenoxy substituents onto the azaphthalocyanine ring.

$\mathrm{UV} / \mathrm{Vis}^{30}$ and ${ }^{1} \mathrm{H} \mathrm{NMR}^{31}$ spectroscopy are powerful techniques used to study aggregation in solution. The UV/Vis absorption spectra of metal ion-containing azaphthalocyanines 2 and 3 $\left(\mathrm{Zn}^{+2}, \mathrm{Co}^{+2}\right)$ in THF solution exhibit a single Q-band accompanied by the typical vibrational satellite band on the blue side, suggesting a $\mathrm{D}_{4 h}$ symmetry for the porphyrazine core (Table 1).

Table 1. Comparison of the Q-band position (nm) displayed by AzaPc 2 and AzaPc 3 in THF with that of its spin coated film

\begin{tabular}{cccc}
\hline AzaPcs & $\mathrm{Q}_{\max }(\mathrm{THF})$ & $\mathrm{Q}_{\max }($ film $)$ & Q shift \\
\hline 2 & 630 & 628 & -2 \\
3 & 618 & 616 & -2 \\
\hline
\end{tabular}

The characteristic Q band has been efficiently used as a probe in assessing the self-assembly features of azaphthalocyanine in solution. Usually, aggregation can be detected by the broadening and blue shift of the Q-band with decrease in intensity. ${ }^{32-35}$ As predicted, the spectra showed monomeric behavior evidenced by a single (narrow) Q-band, typical of metallated 
azaphthalocyanine complexes for both AzaPc 2 and 3 in THF. For example, the absorption spectrum of AzaPc 2 showed a signal sharp Q-band at $\lambda_{\max }=630 \mathrm{~nm}$ (Table 1, Figure 2) which is typical of non-aggregated species. For further assessment, we recorded the absorption spectra for each derivative at different concentrations ranging from $4.6 \times 10^{-6}$ to $11 \times 10^{-6} \mathrm{M}$ as depicted in Figure 2. The position and appearance of the Q-band absorption maxima of AzaPc 2 remained unchanged and consistent with reported spectra of non-aggregated AzaPcs ${ }^{36}$ as the concentration was increased which also results in the increase of the intensity. Similar behavior was detected for AzaPc 3 as outlined in Figure 3. In addition, the apparent molar extinction coefficient (absorptivity) remains constant (Figure 4); this further emphasizes nonaggregation in this concentration range.

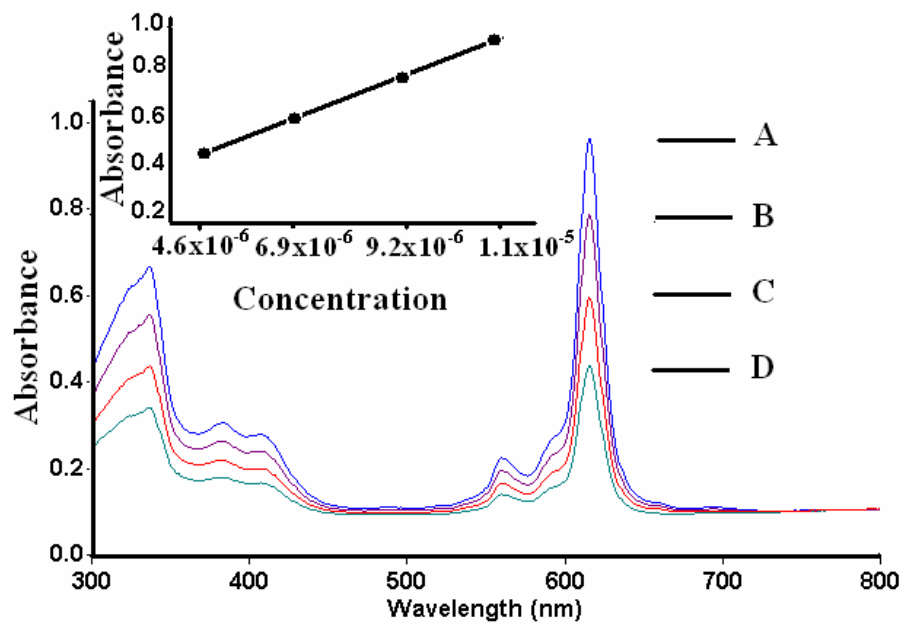

Figure 2. Self- association behavior of $\mathrm{ZnAzaPc}(2)$ in THF at different concentrations: $11 \times 10^{-6}$ (A), $9.2 \times 10^{-6}(\mathrm{~B}), 6.9 \times 10^{-6}(\mathrm{C}), 4.6 \times 10^{-6}(\mathrm{D}) \mathrm{M}$.

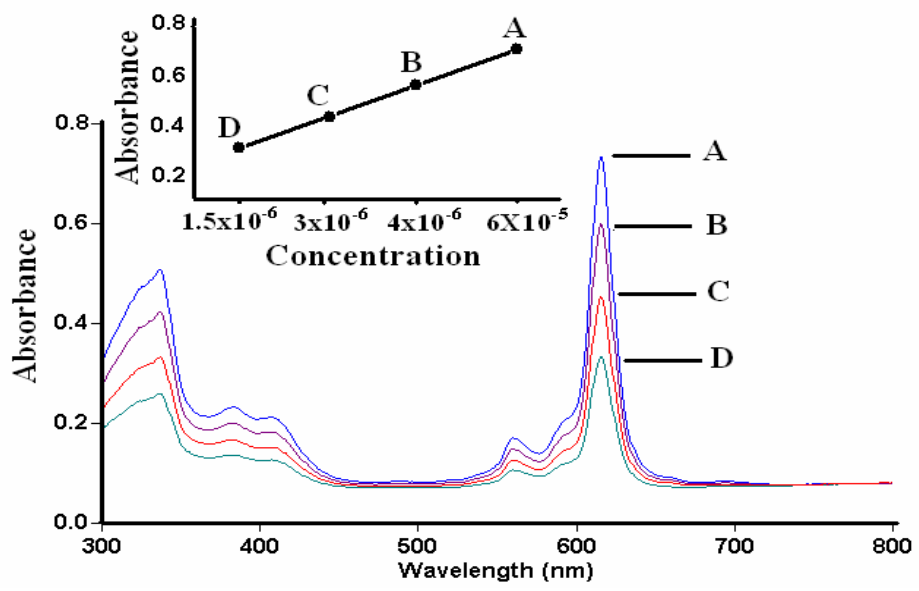

Figure 3. Self- association behavior of AzaPc 3 in THF at different concentrations: $5 \times 10^{-6}(\mathrm{~A})$, $4 \times 10^{-6}(\mathrm{~B}), 3 \times 10^{-6}(\mathrm{C}), 1.59 \times 10^{-6}(\mathrm{D}) \mathrm{M}$. 


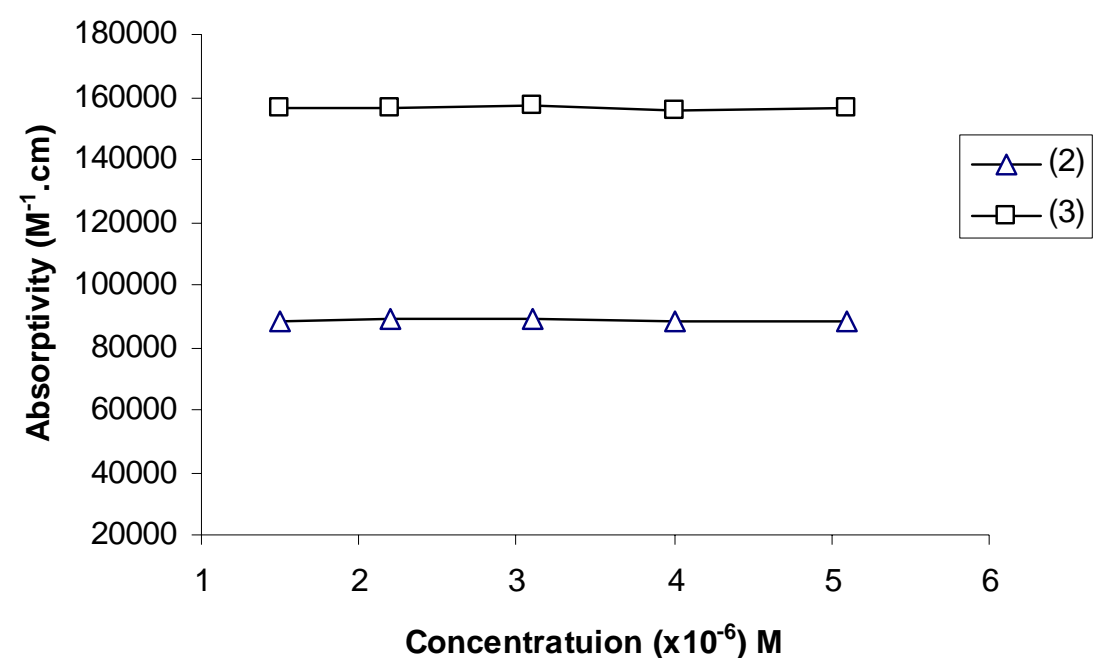

Figure 4. Molar absorption coefficients at the Q-band maxima as a function of concentration for AzaPc complexes (2 and 3 ) in THF.

Controlling the molecular packing within the solid state of an azaphthalocyanine compound is necessary so that the electronic and optical properties (colour, molecular association, optical absorption) can be tailored to the requirements of the intended applications, such as optical limiting devices. Therefore, thin films of complexes $\mathbf{2}$ and $\mathbf{3}$ were prepared to investigate the nature of the azaphthalocyanine derivatives in the solid state. Spin-coated films derived from 2 and 3 were deposited onto glass substrates from chloroform solution. Both complexes gave highly uniform and visually clear films. The UV/visible spectra of spin-coated films (Figure 5, Table 1) of both complexes are almost identical to those obtained from dilute solution spectra, indicating that the AzaPc chromophores are not perturbed by intermolecular exciton effects even in the solid state. The high quality of these films and their intense fluorescence might be useful for device fabrication (e.g. sensors or organic light emitting diodes). This suggests an approach reducing the adverse effects of aggregation when using AzaPc derivatives as photosensitizers in PDT. ${ }^{9}$

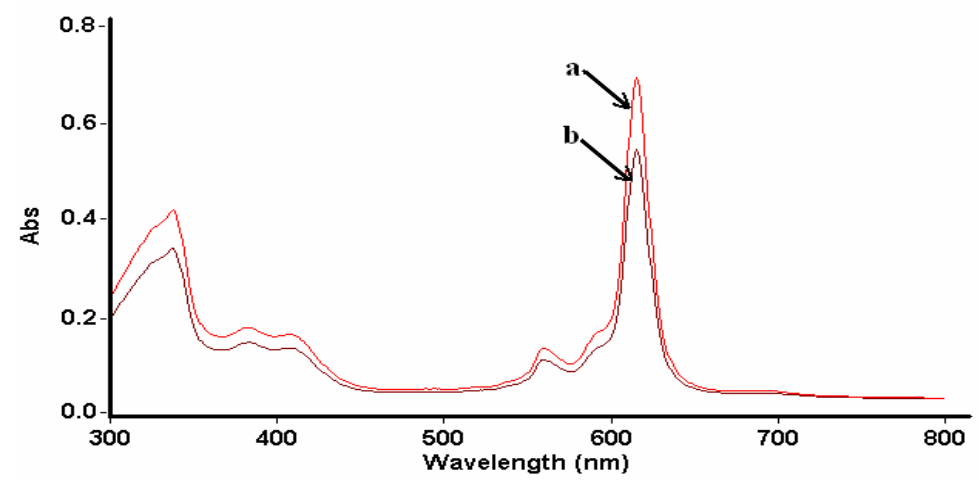

Figure 5. UV/Visible absorption spectrum of AzaPc 2 (a) in THF and (b) from a solvent cast film. 
A commonly observed feature of the ${ }^{1} \mathrm{H}$ NMR spectra of the phthalocyanines and related disc-like molecules such as azaphthalocyanines, is the broadening and upfield shifted peaks due to the self-association of the macrocycle units. ${ }^{37}$ Thus, a good ${ }^{1} \mathrm{H}$ NMR spectrum can be obtained by running the NMR experiment either at high temperature (e.g. $\mathrm{C}_{6} \mathrm{D}_{6}, 60{ }^{\circ} \mathrm{C}$ ) or using $\mathrm{d}_{5^{-}}$ pyridine as solvent to reduce the aggregation of the macrocycle. Therefore, we investigated the aggregation behavior of complexes 2 and 3 by NMR spectroscopy in chloroform over the concentration range between $1 \times 10^{-4}$ and $1 \times 10^{-2} \mathrm{M}$ (Figure 6), at room temperature. Both complexes gave very clean spectra with well-resolved peaks in both the aromatic and aliphatic regions compared to those of reported substituted AzaPc derivatives. In addition, the chemical shifts of the aromatic and aliphatic protons were essentially independent of the concentration as shown in Figure 6. This is most likely attributable to the steric hindrance imposed by the bulky phenoxy substituents that prevent efficient $\pi-\pi$ stacking. Based on the above findings, we emphasise that the position adopted by the bulky substituents relative to the AzaPc core is the most important factor influencing the molecular packing of the AzaPcs in the solid state.

[A] $1 \times 10^{-2} \mathrm{M}$

[B] $5 \times 10^{-3} \mathrm{M}$

[C] $2.5 \times 10^{-3} \mathrm{M}$

D] $1.25 \times 10^{-3} \mathrm{M}$

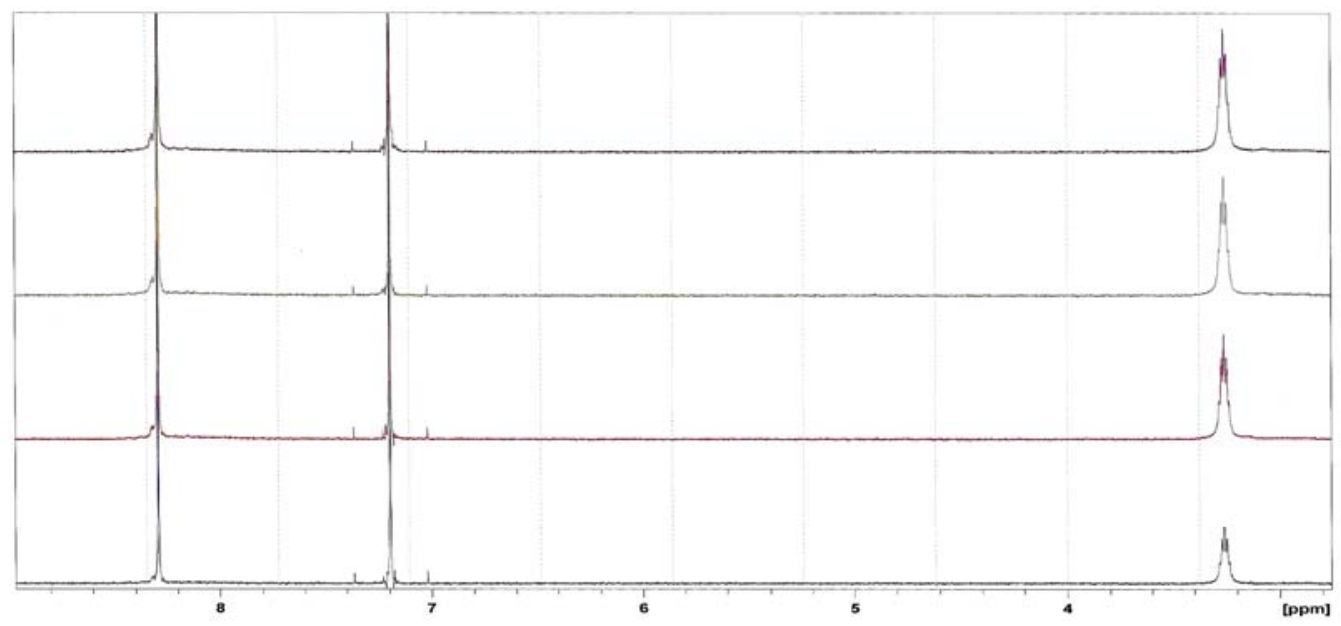

Figure 6. ${ }^{1} \mathrm{H}$ NMR spectra of $\mathrm{AzaPc} 2$ in $\mathrm{CDCl}_{3}$ at room temperature at different concentrations.

\section{Conclusions}

In conclusion, we have successfully synthesized and characterized novel metal-containing octaazaphthalocyanine derivatives using metal-template cyclotetramerisation reactions. Our results showed that the eight bulky substituents represent an efficient way to prevent the aggregation and consequently obtain azaphthalocyanine material with intrinsically true solid solution properties, as inferred by UV/visible and ${ }^{1} \mathrm{H}$ NMR spectroscopy. Due to their interesting properties: the non-aggregation behavior, high solubility, short synthesis and good photostability, 
these new AzaPc derivatives could find applications in organic electronic devices and might be potentially suitable as photosensitizers in PDT.

\section{Experimental Section}

General Procedures. UV-vis spectra were recorded on a Cary 5 spectrophotometer. IR spectra were recorded as KBr pellets using a Perkin Elmer System 2000 FT-IR spectrophotometer. ${ }^{1} \mathrm{H}$ NMR spectra were recorded on a Bruker DPX 400, $400 \mathrm{MHz}$ super-conducting NMR spectrometer. Mass spectra were recorded on a VG Auto-spec-Q (high resolution, high performance, tri-sector GC/MS/MS). The $\mathrm{C}, \mathrm{H}, \mathrm{N}$ analysis of the compounds was performed on a LECO CHNS-932 Elemental Analyzer.

The starting materials, 5,6-dichloro-pyrazine-2,3-dicarbonitrile and 2,6-diisopropyl-4nitrophenol, were prepared following literature procedures. ${ }^{23,24}$

Synthesis of the 5,6-bis(2,6-diisopropyl-4-nitrophenoxy)pyrazine-2,3-dicarbonitrile (1). 2,6diisopropylphenol-4-nitrophenol (4.683 g, $21 \mathrm{mmol})$ was dissolved in $200 \mathrm{ml}$ of dry dioxane containing $\mathrm{NaH}(1.26 \mathrm{~g}, 52.5 \mathrm{mmol})$. Then the reaction mixture was stirred in an ice bath for $2 \mathrm{~h}$, then the 5,6-dichloropyrazine-2,3-dicarbonitrile (2 $\mathrm{g}, 10.1 \mathrm{mmol})$ was added. The reaction mixture was stirred at $100{ }^{\circ} \mathrm{C}$ under nitrogen for $24 \mathrm{~h}$. On cooling, the reaction mixture was poured into $500 \mathrm{ml}$ of distilled water, the resulting precipitate was collected by filtration and recrystallized from diisopropyl alcohol as yellow crystals $(4.86 \mathrm{~g}, 85 \%)$. Mp: $238{ }^{\circ} \mathrm{C}$. Anal. calcd for $\mathrm{C}_{30} \mathrm{H}_{32} \mathrm{~N}_{6} \mathrm{O}_{6}$ : C, 62.87; H, 5.59; N, 14.68\% Found: C, 62.78; H, 5.72; N, 15.02\% MS m/z 573 $(\mathrm{M}+1)^{+}$requires 572. ${ }^{1} \mathrm{H} \mathrm{NMR}\left(\mathrm{CDCl}_{3}\right) \delta \mathrm{ppm}: 1.31(\mathrm{~d}, J=6.2 \mathrm{~Hz}, 24 \mathrm{H}), 2.87(\mathrm{~m}, 4 \mathrm{H}), 8.21(\mathrm{~s}$, 4H). IR (KBr) cm ${ }^{-1}: 1570,1458,1420,1362(\mathrm{C}=\mathrm{C}, \mathrm{C}=\mathrm{N}), 2230(\mathrm{CN})$.

Synthesis of 2,3,9,10,16,17,23,24-octa-5,6-bis(2,6-diisopropyl-4-nitrophenoxy)-1,4,8,11,15, 18,22,25-octaazaphthalocyaninato zinc(II) AzaPc (2). A mixture of 5,6-bis(2,6-diisopropyl-4nitrophenoxy)pyrazine-2,3-dicarbonitrile (1) (2 g, $3.49 \mathrm{mmol})$ and anhydrous zinc acetate $(0.73$ $\mathrm{g}, 3.99 \mathrm{mmol})$ in dry quinoline $(80 \mathrm{ml})$ was stirred at $160{ }^{\circ} \mathrm{C}$ for $24 \mathrm{~h}$ under nitrogen. On cooling, the reaction mixture was poured into $200 \mathrm{ml}$ distilled water, the formed solid product was purified by column chromatography using a mixed solvent (hexane: ethyl acetate 1:1) as eluent and recrystallised from acetone as a green solid (1.15 g, 49\%). Mp: $>300{ }^{\circ} \mathrm{C}$. Anal. calcd for $\mathrm{C}_{120} \mathrm{H}_{128} \mathrm{~N}_{24} \mathrm{O}_{24} \mathrm{Zn}$ : C, 61,17; H, 5.43; N, 14.27\% Found: C, 60.77; H, 5.29; N, 13.84\% MS m/z (MALDI) 2354. UV: $\lambda_{\max } \mathrm{nm}$ (THF) (log $\varepsilon$ ): 369 (4.802), 575 (4.538), 630 (4.95). ${ }^{1} \mathrm{H}$ NMR $\left(\mathrm{CDCl}_{3}\right) \delta \mathrm{ppm}: 1.34(\mathrm{~d}, J=7.2 \mathrm{~Hz}, 96 \mathrm{H}), 3.36(\mathrm{~m}, 16 \mathrm{H}), 8.38(\mathrm{~s}, 16 \mathrm{H}) . \mathrm{IR}(\mathrm{KBr}) \mathrm{cm}^{-1}: 1575$, $1450,1418,1369(\mathrm{C}=\mathrm{C}, \mathrm{C}=\mathrm{N})$.

Synthesis of 2,3,9,10,16,17,23,24-octa-5,6-bis(2,6-diisopropyl-4-nitrophenoxy)-1,4,8,11,15, 18,22,25-octaazaphthalocyaninato cobalt(II) AzaPc (3). AzaPc 3 was prepared from 1 by a similar procedure to that for AzaPc 2 and purified by column chromatography using a mixed solvent (hexane: ethyl acetate 1:1) as eluent and recrystallised from acetone as a blue solid (1.18 
g, 49\%). Mp: $>300{ }^{\circ} \mathrm{C}$. Anal. calcd for $\mathrm{C}_{120} \mathrm{H}_{128} \mathrm{~N}_{24} \mathrm{O}_{24} \mathrm{Co}: \mathrm{C}, 61,35 ; \mathrm{H}, 5.45 ; \mathrm{N}, 14.30 \%$ Found: C, 60.96; H, 5.29; N, 13.91\% MS m/z (MALDI) 2347. UV: $\lambda_{\max }$ nm (THF) (log $\left.\varepsilon\right): 349$ (5.201), 618 (5.134). ${ }^{1} \mathrm{H}$ NMR $\left(\mathrm{CDCl}_{3}\right) \delta$ ppm: 2.28 (d, $\left.J=6.2 \mathrm{~Hz}, 96 \mathrm{H}\right), 5.09(\mathrm{~m}, 16 \mathrm{H}), 9.32(\mathrm{~s}, 16 \mathrm{H})$. IR $(\mathrm{KBr}) \mathrm{cm}^{-1}: 1570,1456,1422,1369(\mathrm{C}=\mathrm{C}, \mathrm{C}=\mathrm{N})$.

\section{Acknowledgements}

We are grateful for financial support from the University of Kuwait received through Research Grant No SC08/02 and the facilities used from ANALAB and SAF.

\section{References and Notes}

1. Mckeown, N. B. Phthalocyanine Materials: synthesis structure and function; CUP: Cambridge, 1998.

2. Leznoff, C. C.; Lever, A. B. P. Phthalocyanines: Properties and Applications; VCH: New York, 1989, 1993, 1996; Vol. 1-4.

3. de la Torre, G.; Claessens, C. G.; Torres, T. Chem. Commun. 2007, 2000.

4. McKeown, N. B. J. Mater. Chem. 2000, 10, 1979.

5. Kudrevich, S. V.; Van Lier, J. E. Coord. Chem. Rev. 1996, 156, 163.

6. Ghosh, A.; Gassman, P. G.; Almlof, J. J. Am. Chem. Soc. 1994, 116, 1932.

7. Gal'pern, M. G.; van Lier, J.E. Synthesis 1994, 779.

8. Zimcik, P.; Miletin, M.; Kostka, M.; Schwarz, J.; Musil, Z.; Kopecky, K. J. Photochem. Photobiol., A 2004, 163, 21.

9. Kosta, M.; Zimcik, P.; Miletin, M.; Klemera, P.; Kopecky, K.; Musil, Z. J. Photobiol., A 2006, 178, 16.

10. Lee, B. H.; Jaung, J. Y.; Jang, S. C.; Yi, S. C. Dyes Pigments. 2005, 65, 159.

11. Maizish, V. E.; Korzheneveskii, A. B.; Klyuev, V. N. Khim. Geterotsikl. Soedin. 1984, 1257.

12. Ohta, K.; Watanable, T.; Hasebe, H.; Morizumi, Y.; Fujimoto, T.; Yamamoto, I.; Lelivever, D.; Simon. J. Mol. Cryst. Liq. Cryst. 1991, 1611.

13. Ohta, K.; Watanabe, T.; Fujimoto, T.; Yamamoto, I. Chem. Commun. 1989, 1611.

14. Mohr, B.; Wegner, G.; Ohta, K. J. Chem. Commun. 1995, 995.

15. Wen, T. C.; Chen, S. P.; Tsai, C. Y. Synthetic Met. 1998, 97, 105.

16. Jaung, J. Y.; Matsuoka, M.; Fukunishi, K. Dyes Pigments 1999, 40, 73.

17. Musil, Z.; Zimcik, P.; Miletin, M.; Kopecky, K.; Petrik, P.; Lenco, J. Photochem. Photobiol., A 2007, 186, 316.

18. Zimcik, P.; Miletin, M.; Ponec, J.; Kostka, M.; Fiedler, Z. J. Photochem. Photobiol. B Biol. 2000, 20. 
19. Miroslav, K.; Petr, Z.; Miroslav, M. J. Photochem. Photobiol. B Biol. 2006, 16.

20. Miroslav, K.; Petr, Z.; Miroslav, M. J. Photochem. Photobiol. B Biol. 2003, 127.

21. Mckeown, N. B., in: Kadish, K. M., Smith, K. M. \& Guilard, R. D. (Eds.). The Porphyrin Handbook. Academic Press: New York. 2003, 15, 61-123.

22. Mckeown, N. B. In Science of Synthesis; Weinreib, S. Ed.; Thieme: Stuttgart, 2004; 1258.

23. Suzuki, T.; Nagae, Y.; Mitsuhashi, K. J. Heterocycl. Chem. 1986, 194.

24. Trapani, G.; Latrofa, A.; Franco, M.; Altomare, C. J. Med. Chem. 1998, 41, 1846.

25. Costanza, B.; Maria, P.; Claudio, E.; Fabrizio, M. Inorg. Chem. 2005, 44, 9852

26. Stuzhin, P.; Ercolani, C.; The Porphyrin Handbook; Academic Press: New York. 2003, 15, Chapter 101, 263-364.

27. (a) Yanagi, H.; Wada, M.; Ueda, Y.; Ashida, M. Makromol. Chem. 1992, 193, 1903. (b) Yakushi, M.; Shirotani, I.; Khairullin, I. I.; Nakazawa, Y.; Kanoda, K.; Kosugi, N.; Takeda, S. Synthetic Met. 1995, 71, 2287.

28. (a) Yudasaka, M.; Nakanishi, K.; Hara, T.; Tanaka, M.; Kurita, S. J. Appl. Phys. 1985, 24, 887. (b) Cherkashina, L. G.; Berlin, A. A. Vysokomol. Soedin. 1966, 8, 627.

29. (a) Xu, H-T.; Xie, W-W.; Guo, Y-C.; Ci, Y.; Chen, L-J.; Peng, B-X. J. Chinese Chem. Soc. 2007, 54, 211. (b) Kobayashi, N.; Nakajima, S.; Osa, T. Inorg. Chem. Acta 1994, 116, 879.

30. (a) Law, W.-F.; Lui, K. M.; Ng, D. K. P. J. Mater. Chem. 1997, 7, 2063. (b) Tai, S.; Hayashi, N. J. Chem. Soc., Perkin Trans. 2 1991, 1275.

31. Chen, M. J.; Rathke, J. W. J. Porphyrin Phthalocyanines 2001, 5, 528.

32. (a) Ahsen, V.; Yilmazer, E.; Gürek, A. G.; Gül, A.; Bekaroğlu, Ö. Helv. Chim. Acta 1988, 71, 1988. (b) Stillman, M. J.; Nyokong, T. Phthalocyanines Properties and Applications, Ed. Leznoff, C. C.; A. B. P., Lever. VCH: Weinheim. 1989; Vol 1, 133.

33. (a) Okur, A. I.; Gül, A.; Cihan, A.; Tan, N.; Bekaroğlu, Ö. Synth. React., Inorg. Met.-Org. Chem. 1990, 20, 1399. (b) Piechocki, C.; Simon J. Nouv. J. Chem. 1985, 9, 159.

34. (a) Hendriks, R.; Sielcken, O. E.; Drenth, W. J. Chem. Soc., Chem. Commun. 1986, 1464. (b) Sielcken, O. E.; van Tilborg, M. M.; Roks, M. F. M.; Hendriks, R.; Drenth, W.; Nolte, R. J. M. J. Am. Chem. Soc. 1987, 109, 4261.

35. (a) Kobayashi, N.; Nishiyama, Y. J. Chem. Soc., Chem. Commun. 1986, 1462. (b) Kobayashi, N.; Lever, A. B. P. J. Am. Chem. Soc. 1987, 109, 7433.

36. Makhseed. S.; Ibrahim, F.; Bezzu, C. G.; Mckeown, N. B. Tetrahedron Lett. 2007. (Accepted).

37. Makhseed, S.; Mckeown, N. B; Msayib, K.; Bumajdad, A. J. Materials Chem. 2005, 15, 1. 